\title{
Electrophysiologic characteristics of tremor in Parkinson's disease and essential tremor
}

\author{
Características eletrofisiológicas do tremor na Doença de Parkinson e tremor essencial
}

Ederson Cichaczewski', Renato P. Munhoz², Joaquim M. Maia', Percy Nohama', Edison M. Nóvak ${ }^{3}$. Helio A. Teive

\begin{abstract}
Tremor in essential tremor (ET) and Parkinson's disease (PD) usually present specific electrophysiologic profiles, however amplitude and frequency may have wide variations. Objective: To present the electrophysiologic findings in PD and ET. Method: Patients were assessed at rest, with posture and action. Seventeen patients with ET and 62 with PD were included. PD cases were clustered into three groups: predominant rest tremor; tremor with similar intensity at rest, posture and during kinetic task; and predominant kinetic tremor. Results: Patients with PD presented tremors with average frequency of $5.29 \pm 1.18 \mathrm{~Hz}$ at rest, $5.79 \pm 1.39 \mathrm{~Hz}$ with posture and $6.48 \pm 1.34 \mathrm{~Hz}$ with the kinetic task. Tremor in ET presented with an average frequency of $5.97 \pm 1.1 \mathrm{~Hz}$ at rest, $6.18 \pm 1 \mathrm{~Hz}$ with posture and $6.53 \pm 1.2 \mathrm{~Hz}$ with kinetic task. Seven (41.2\%) also showed rest tremor. Conclusion: The tremor analysis alone using the methodology described here, is not sufficient to differentiate tremor in ET and PD.
\end{abstract}

Keywords: Parkinson's disease, essential tremor, tremor.

\section{RESUMO}

Os tremores observados no tremor essencial (TE) e na doença de Parkinson (DP) costumam apresentar perfis eletrofisiológicos específicos, embora amplitude e frequência possam ter grandes variações. Objetivo: Apresentar os resultados dos exames eletrofisiológicos na DP e no TE. Método: Pacientes foram avaliados em repouso, com postura e em ação. Foram incluídos 17 pacientes com TE e 62 com DP. Casos de DP foram divididos em três grupos: predomínio de tremor de repouso; tremor com intensidade semelhante em repouso, postura e tarefa cinética e tremor cinético predominante. Resultados: Pacientes com DP apresentaram tremores com

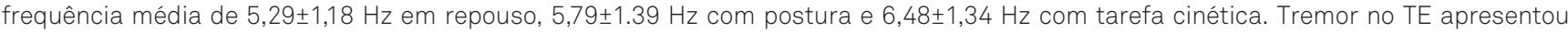

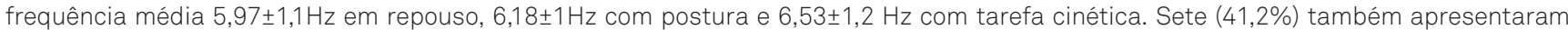
tremor de repouso. Conclusão: A análise do tremor per se, usando os métodos descritos neste estudo, não é suficiente para diferenciar o tremor no TE e DP.

Palavras-chave: doença de Parkinson, tremor essencial, tremor.

Essential tremor (ET) and Parkinson's disease (PD) are two of the most common adult-onset tremor disorders. The prevalence of both increases with age and is estimated to be $1.8 \%$ in individuals who are 65 years of age and older in the case of $\mathrm{PD}$, and $4.6 \%$ in the same age group for $\mathrm{ET}^{1}$. Identification and characterization of different forms of tremor is routine practice for most clinicians, however, it is also a challenging task ${ }^{2}$. Although these tremors are usually identified using subjective parameters from a clinical bedside perspective, from a physiological standpoint they typically have specific characteristics regarding frequencies and amplitude measurements, as well as their correlation with posture (rest versus action) $)^{3}$. In the case of ET, tremor typically occurs predominantly with action and oscillates at a frequency of 8 to $12 \mathrm{~Hz}$, inversely correlating with its severity ${ }^{4}$, while for PD, tremor occurs in almost three fourths of all cases, is more often noticeable at rest, with a frequency of that ranges from 4 to $6 \mathrm{~Hz}^{5}$. These profiles, however, may present with significant overlap, as in the case of patients with ET who present with some degree of rest tremor and parkinsonian patients with re-emergent postural tremor ${ }^{3,5}$. Also, the oscillations of these pathological tremors are only approximately sinusoidal, with both amplitude and frequency presenting with wide variations ${ }^{3}$.

${ }^{1}$ Programa de Pós-Graduação em Engenharia Elétrica e Informática Industrial, Universidade Tecnológica Federal do Paraná, Curitiba PR, Brazil; ${ }^{2}$ Associação Paranaense de Portadores de Parkinsonismo, Curitiba PR, Brazil;

${ }^{3}$ Departamento de Neurologia, Departamento de Medicina Interna, Hospital de Clínicas, Universidade Federal do Paraná, Curitiba PR, Brazil. Correspondence: Dr. Helio A G Teive; Rua General Carneiro 1103/102, Centro; 80060-150 Curitiba PR - Brasil; E-mail: hagteive@mps.com.br Conflict of interest: There is no conflict of interest to declare.

Received 18 July 2013; Received in final form 15 November 2013; Accepted 04 December 2013. 


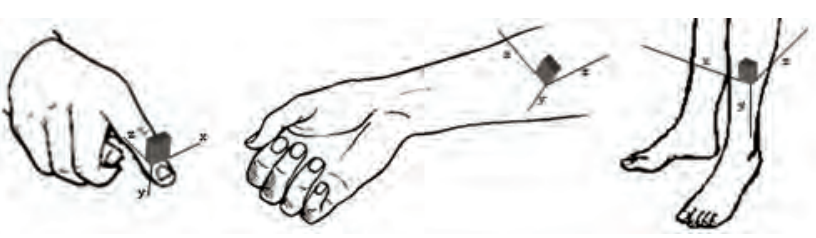

Figure 1. Accelerometer placement for the index finger, forearm and leg.

The objectives of this study were to present the findings obtained with an eight sensor system developed for the measurement of tremor and to analyze its ability to differentiate oscillations in PD and ET.

\section{METHOD}

Cases with an established diagnosis of PD and ET were included in the study. Patients were asked to come for the assessment after skipping the previous dose of medication used to treat ET or dopaminergic treatment, in the case of PD. Therefore, patients were assessed during their "off" medications periods.

The system used for the analysis included sensors (accelerometers) that capture movement in two dimensions. The sites for sensors placement were defined with the assistance of the senior author, specialist in movement disorders. Three were used for each upper limb (one each on the middle finger, index finger, forearm) and one for each lower limb (anterior aspect of the tibia), leaving the measuring axes perpendicular to the longitudinal axis of the limb, totaling eight sensors (Figure 1). To prove the effectiveness of the measurements, the system was calibrated statically and dynamically twice to verify repeatability; the correlation coefficient obtained was $\mathrm{r}^{2}=0.99$ for all axes. Measurement system frequencies ranged from 0 to $30 \mathrm{~Hz}$. Sensors were electrically isolated from their power source and communication lines, as specified by NBR IEC 601-1. The analysis used a Freescale MMA6260Q Series: X-Y axis micromachined accelerometer (Chandler, AZ) and software from Crossbow Inc. The sample frequency rate was set to $50 \mathrm{~Hz}$ per channel. We used high pass analogical filters to guarantee DC block and anti-aliasing low pass filters to allow dynamic signal bandwidth. Frequency was properly attenuation at $280 \mathrm{~Hz}$, which is the frequency of the sampled signals. allowing enough signal to noise ratio for the measurement accuracy of the signals by the A/D converter. Later, the software applies a FIR low pass filter to produce an attenuation of - $60 \mathrm{~dB}$ when the signal reaches $60 \mathrm{~Hz}$, with a cutoff frequency of $20 \mathrm{~Hz}$.

The analyses were performed to determine specific features of each disorder in regards to movements' amplitude and frequency. The peak frequency was computed using power spectral density (PSD) calculations, reporting the peak frequency as well as the amplitude at this peak frequency (g). Additionally, root mean square (RMS) amplitude, which is equivalent of the mean value of the waveform after rectification, of the 50 second epoch was computed.

The project was approved by the local ethics committee and all patients involved provided a signed informed consent.

Patients were recruited from the outpatient clinics of the Movement Disorders service, Hospital de Clínicas, Universidade Federal do Paraná and the Associação Paranaense de Portadores de Parkinsonismo. Patients were excluded if they could not tolerate been "off" medication or had a history of: current use of medications know to cause tremor, previous stereotactic surgery and any other neurologic condition that interfered with tremor phenotype.

Patients were assessed with sensors placed as previously described, at rest, with limbs fully extended (postural task), and, for the upper limbs, a kinetic task (finger to nose movement) as shown in Figure 2. Rest tremor was assessed with the upper limbs in a resting position, supported against gravity, comfortably placed on the subject's thighs, while repeatedly reminded not to move or activate muscles of the upper limbs and shoulders. Data collected from the most affected side was used for the analysis. Measurements lasted from 20
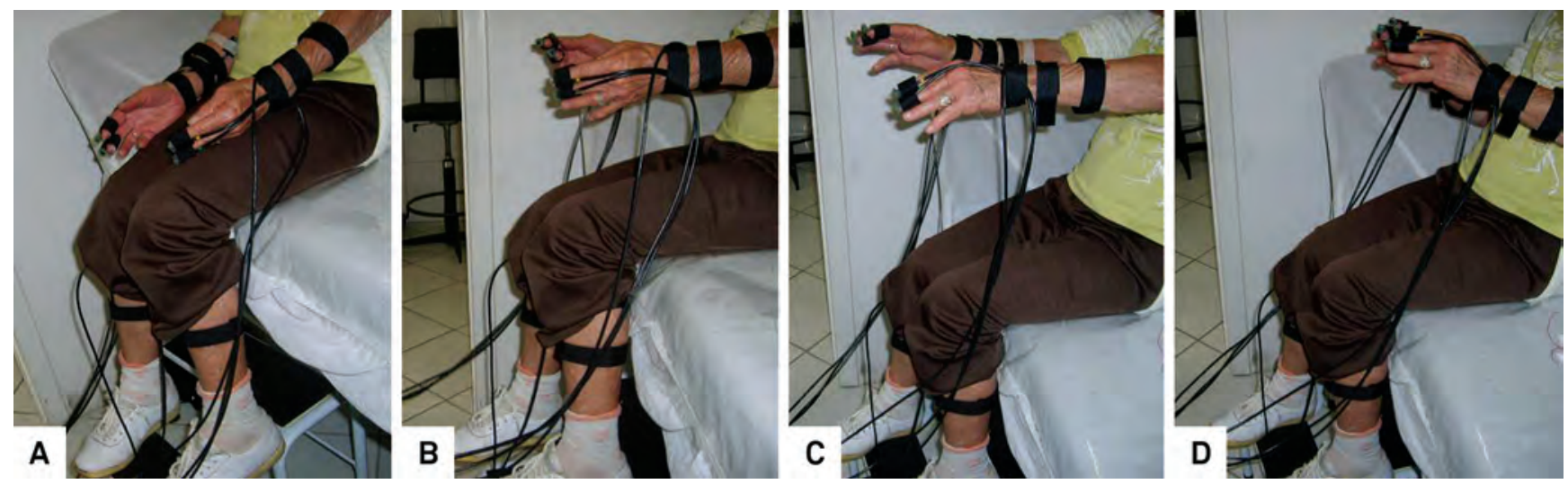

Figure 2. Testings for rest (A), postural (B) and kinetic (C,D) tremors. 
Table 1. Comparison of tremor frequency between cases of Parkinson's disease (PD) and essential tremor (ET) on rest and action tasks.

\begin{tabular}{lccc} 
Mean tremor frequency $(\mathrm{Hz})$ & $\mathrm{PD}$ & $\mathrm{ET}$ & $\mathrm{p}$-value* \\
\hline Average & $5.8 \pm 1.3$ & $6.2 \pm 1.2$ & 0.2 \\
Rest & $5.3 \pm 1.2$ & $6 \pm 1.1$ & 0.11 \\
Postural & $5.8 \pm 1.4$ & $6.2 \pm 1$ & 0.19 \\
Kinetic & $6.5 \pm 1.3$ & $6.5 \pm 1.2$ & 0.7 \\
\hline
\end{tabular}

*Mann-Whitney U-test.

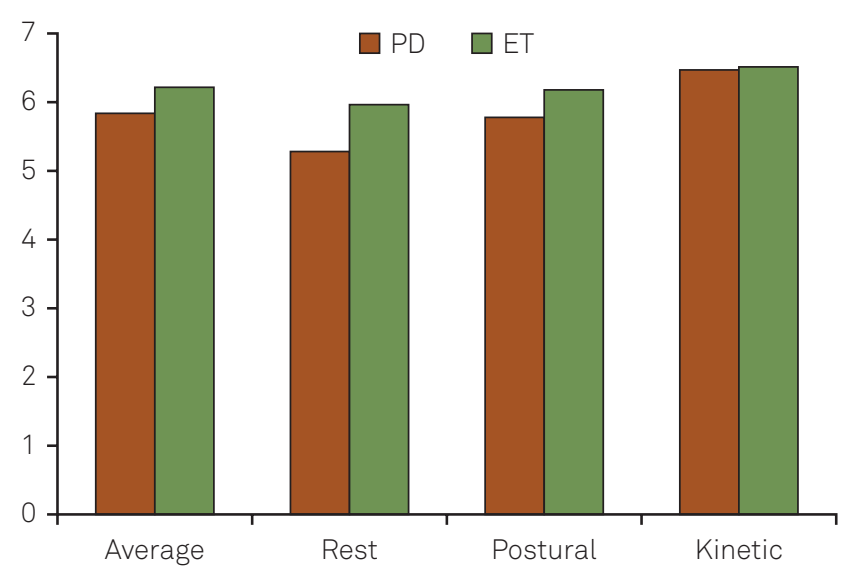

Y axis represents frequency in $\mathrm{Hz}$. P-value for all comparisons $>0.05$.

Figure 3. Graphic representation of mean tremor frequencies for Parkinson's disease (PD) and essential tremor (ET) patients.

to 30 minutes. In the case of PD, all cases were assessed in the practical OFF condition and disease severity was determined using the Hoehn and Yahr staging scale $(\mathrm{H} \& \mathrm{Y})^{6}$. Diagnoses of ET and PD were based on validated criteria. For PD, the Queen Square Brain Bank criteria, that requires the presence of rigidity in addition to any of the other three cardinal signs (rest tremor, bradykinesia and postural instability) and the absence of atypical features that can indicate alternative diagnoses. For ET, the presence of bilateral, largely symmetric postural or kinetic tremor involving the head and forearms, in the absence of abnormal postur- ing, and excluding other known forms of secondary or primary tremor ${ }^{7,8}$.

Mann-Whitney U-test was used to establish statistically significant associations. P-value of less than 0.05 was considered statistically significant. Additionally, to determine the average of the measurements and extreme limits, were used the arithmetic mean and standard deviation, respectively.

\section{RESULTS}

A total of 17 patients with ET (mean age $62.3 \pm 18$ years, 11 women) and 62 patients with PD (mean age $65.7 \pm 12.1$ years, 32 women) were included in the study.

A comparison of tremor frequencies in cases of PD and ET are shown in Table 1 and Figure 3.

As a whole, patients with PD presented tremors with average frequency of $5.29 \pm 1.18 \mathrm{~Hz}$ at rest, $5.79 \pm 1.39 \mathrm{~Hz}$ with posture and $6.48 \pm 1.34 \mathrm{~Hz}$ during the kinetic task with an average among the three tests of $5.85 \pm 1.31 \mathrm{~Hz}$, and a $95 \%$ confidence that the frequency of tremors lies between $5.53 \mathrm{~Hz}$ and $6.18 \mathrm{~Hz}$, using normal distribution probabilities. Lower limb tremor was detected in 10 (16.1\%) cases using the above mentioned methods.

A more detailed analysis of the results found in PD cases showed that patients could be grouped into three distinct clusters (Table 2): subgroup 1: presenting predominant rest tremor in regards to movement intensity (13 patients, $20.1 \%$ ); subgroup 2: presenting tremor with similar intensity at rest, posture and during kinetic task (27 patients, 43.6\%); subgroup 3: presenting more intense kinetic tremor (22 patients, 36.3\%). In summary, the RMS amplitude of tremor allowed division of cases of PD into the three above mentioned subgroups, subgroup 1 with the characteristics of a more pure rest tremor and subgroup 3 with characteristics quite similar to those of ET. These three subgroups were, however, not significantly different regarding demographic variables and disease severity.

Table 2. Demographic, clinical and tremor measurement data collected from patients with Parkinson's disease, divided according to subgroups.

\begin{tabular}{lccc}
\hline & Subgroup 1 & Subgroup 2 & Subgroup 3 \\
\hline Number of cases (\%) & $13(20.1 \%)$ & $27(43.6 \%)$ & $22(36.3 \%)$ \\
Gender men (\% within group) & $6(46.1 \%)$ & $13(48.1 \%)$ & $11(50 \%)$ \\
Mean age \pm SD (years) & $69 \pm 10.1(43-81)$ & $63.9 \pm 12(39-87)$ & $65.1 \pm 14.2(21-84)$ \\
Disease duration \pm SD (years) & $6.7 \pm 4.5$ & $9 \pm 6.1$ & $8.2 \pm 5$ \\
Mean H\&Y stage \pm SD & $3.2 \pm 0.3$ & $3.1 \pm 0.2$ & $2.8 \pm 0.6$ \\
Mean frequency rest (Hz) & $5.5 \pm 1.3$ & $5.3 \pm 1.4$ & $6 \pm 1.5$ \\
Mean RMS rest (g) & $0.047 / 0.018$ & $0.046 / 0.08$ & $0.023 / 0.012$ \\
Mean frequency posture $(\mathrm{Hz})$ & $5.5 \pm 1.2$ & $5.5 \pm 1.6$ & $6.1 \pm 0.9$ \\
Mean RMS posture $(\mathrm{g})$ & $0.024 / 0.014$ & $0.068 / 0.147$ & $0.026 / 0.016$ \\
Mean frequency kinetic $(\mathrm{Hz})$ & $7.1 \pm 1.6$ & $6.6 \pm 1.7$ & $7.4 \pm 1$ \\
Mean RMS kinetic $(\mathrm{g})$ & $0.024 / 0.011$ & $0.069 / 0.109$ & $0.040 / 0.023$ \\
\hline
\end{tabular}

SD: standard deviation; H\&Y: Hoehn \& Yahr scale; RMS: root mean square. 
Table 3. Demographic, clinical and tremor measurement data collected from patients with ET.

\begin{tabular}{lc} 
Number of cases & $\mathbf{1 7}$ \\
Gender men (\%) & $6(35.3 \%)$ \\
Mean age (years \pm ) & $62.3 \pm 18(17-83)$ \\
Mean disease duration (years) & $13.1 \pm 6.4$ \\
Rest tremor $(\%)$ & $7(41.2 \%)$ \\
Mean frequency $(\mathrm{Hz})$ & $6 \pm 1.1$ \\
Mean RMS rest $(\mathrm{g})$ & $0.025 \pm 0.018$ \\
Mean frequency posture $(\mathrm{Hz})$ & $6.2 \pm 1$ \\
Mean RMS posture $(\mathrm{g})$ & $0.028 \pm 0.020$ \\
Mean frequency kinetic $(\mathrm{Hz})$ & $6.5 \pm 1.2$ \\
Mean RMS kinetic $(\mathrm{g})$ & $0.072 \pm 0.038$ \\
\hline
\end{tabular}

Cases of ET (Table 3) presented tremor with an average frequency of $5.97 \pm 1.1 \mathrm{~Hz}$ at rest, $6.18 \pm 1 \mathrm{~Hz}$ with posture and $6.53 \pm 1.2 \mathrm{~Hz}$ with the kinetic task, with an average among the three tests of $6.22 \pm 1.2 \mathrm{~Hz}$, and a $95 \%$ confidence that the frequency of tremors lies between $5.81 \mathrm{~Hz}$ and $6.63 \mathrm{~Hz}$, using Student's $t$-test. Among these cases, by definition, all patients presented with postural and kinetic tremor, while 7 (41.2\%) also showed rest tremor. In this group, tremor was considered significantly asymmetric in $3(17.6 \%)$ and no patient showed lower limb tremor detected by the sensor analysis. Graphic representations of tremor frequency recordings in PD and ET are shown in Figure 4.

\section{DISCUSSION}

In this study we showed that tremor analysis is not able to individually differentiate cases of ET and PD, especially frequence as a variable. However, when mean values were used, groups showed significant differences. The amplitude RMS profile also showed a similar trend, presenting signific- ant differences only when comparing mean values collected from groups of patients with either ET and PD. Individually, a significant number of ET cases presented with detectable rest tremor, while in cases with $\mathrm{PD}$, amplitude was able to separate patients into three distinct subgroups, one of them (subgroup 3) with characteristics that overlapped those of ET. Therefore, our findings imply that tremor analysis per se, using the methods described here, may not be sufficient for a definitive differential diagnosis. For PD, as in the case of a diagnosis based on clinical grounds, the analysis used here would probably be refined by the addition of data regarding the additional cardinal features (bradykinesia and rigidity) of the disease.

The occurrence of a rest component in cases of ET has been widely recognized in the literature. Cohen et al. ${ }^{9}$, for instance, found rest tremor in the absence of other parkinsonian signs in almost 1 of every 5 patients with ET. In more than half of these, it was present during at least 2 activities (standing or walking and while seated). The authors confirmed the finding electrophysiologically, showing absence of EMG activity in the tremulous upper limb. Rest tremor in ET was detected in cases whose symptom was present for longer, was more severe, and more disseminated. The basis for rest tremor in ET is not clear. If we consider the existence of a correlated pathological process, it would sound convenient to admit that in patients with severe, long-standing, and disseminated disease, the pathology responsible for ET may have spread into motor systems outside of the cerebellum-cerebellar outflow connections, including the basal ganglia and their connections ${ }^{10}$. Another more plausible possibility is that rest tremor in ET is not a true rest tremor but a breakthrough postural tremor, spreading from more proximal body parts, which possibly were not tested in the Cohen et al. ${ }^{9}$ study previously mentioned.
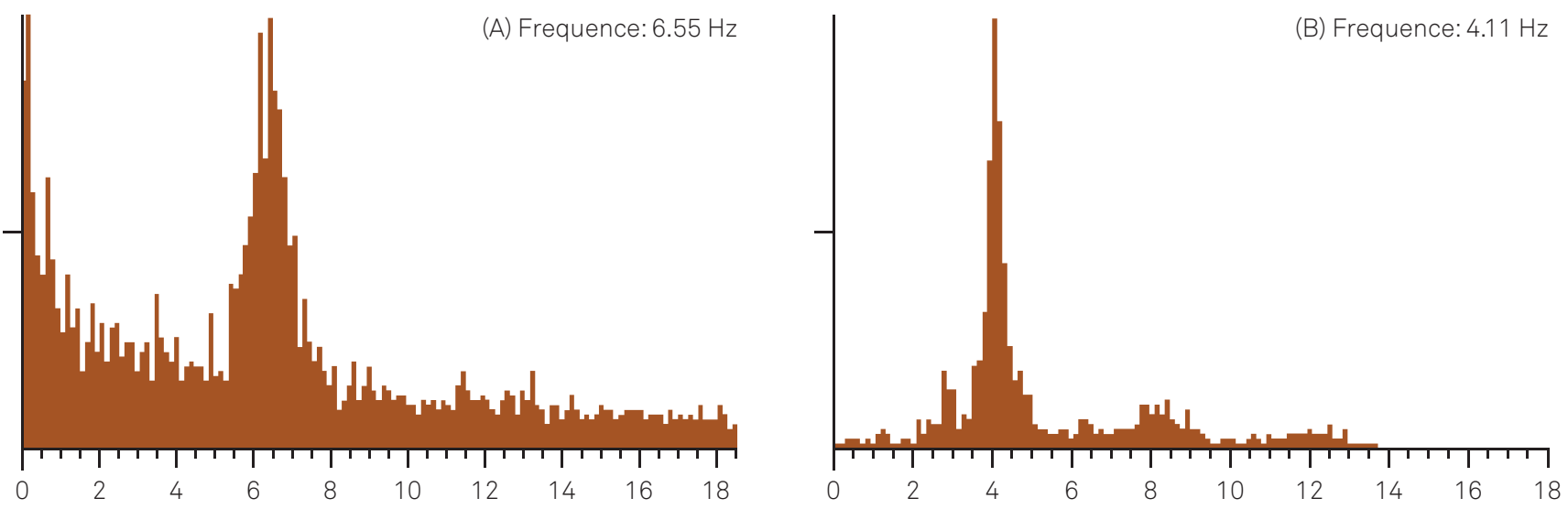

Figure 4. Graphic representations of tremor in a patient with essential tremor (A) during the kinetic task and Parkinson's disease (B) at rest $X$ axis represents frequency. 
In regards to $\mathrm{PD}$, we found that tremor phenotype can be clustered into 3 subgroups. Although we anticipated differences regarding demographic and/or clinical differences among these subgroups, these variables proved to be consistently similar across the whole sample, as suggested by the study of Louis et al. ${ }^{11}$. We also found that tremors that can be electrophysiologically detected with more intensity at rest (subgroup 1) may not be the prototypical tremor in PD. Indeed, most of our cases (subgroup 2, 43.6\%) presented with tremor that had similar intensities during the three postures studied. Concomitance of rest and action tremor is common in PD, with a prevalence as high as $92 \%$. Kraus et al. ${ }^{12}$ studied a large sample of $870 \mathrm{PD}$ patients showing that a combination of rest, postural and kinetic tremors constitute the most frequent tremor phenotype in PD. While some consider this tremor to be an occasional and unimportant finding, in clinical practice, a moderate action tremor may be more dysfunctional than typical parkinsonian rest tremor ${ }^{13}$. As in the case of rest tremor in ET, the basis for action tremor in PD is unclear. Most patients tend to present with an action tremor more pronounced on the side of the body that is more affected by the other signs of parkinsonism ${ }^{14}$. Also, action tremor may respond to the same interventions used to treat its rest correlate ${ }^{15}$, suggesting a similar underlying pathological basis. On the other hand, tremor analysis of cases of action tremor in PD showed that some cases present findings similar to exacerbated physiological tremor ${ }^{14,15}$.

Finally, subgroup 3 presented with predominant kinetic tremor. This form of tremor in PD typically occurs with a measured frequency that may vary between 4 and $9 \mathrm{~Hz}$. It has been more consistently described in the akinetic rigid variant of $\mathrm{PD}$, which is probably the case in our sample. Some cases may alternatively be caused by a combination of incidental ET or exacerbated physiological tremor among cases of rigid akinetic $\mathrm{PD}^{16}$.

These findings may have clinical implications from diagnostic and prognostic points of view. First, it shows that tremor in PD can be manifested in different patterns, not confined to the characteristic rest predominant oscillation. Therefore, the lack of the archetypal pill rolling tremor in a patient with otherwise typical parkinsonism, should not be a reason to question $\mathrm{PD}$ as final diagnosis. Also, during the last decades, several studies showed that PD patients with the most typical presentation of pure resting tremor have not only a more benign disease progression from the motor standpoint, but also a lower incidence of axial motor and non-motor features. These features are, in most cases, treatment refractory and have a very significant impact in functionality and quality of life in more advanced stages. Our study was not designed to assess these potential implications, therefore, these are mere conjectures ${ }^{17}$. Future longitudinal studies may help to address these issues.
The physiopathology of tremor in PD is complex and cannot be explained simply by changes in neurotransmitter concentrations and activity in the basal ganglia, as in the case of rigidity, bradykinesia and postural instability. Tremor in these cases is possibly generated by groups of neurons in the basal ganglia which act as central oscillators, producing repetitive impulses to the involved body parts. It is also influenced by peripheral neural structures, which exert modulatory influence on amplitude. Therefore, rest tremor in PD is the result of increased activity and synchronization of central oscillators ${ }^{18}$. Changes in tremor amplitude and frequency are attributed to variability in the activity and synchronization of central oscillators in the basal ganglia; these changes are probably demonstrated during typical examination paradigms (for example, arm rest and action tasks). In addition, the recurrence of clinically visible rest tremor is accompanied by a reduction in tremor frequency variability. This reduction is attributed to increased synchronization of central oscillators in basal ganglia. Both mechanisms are equally important and typically tremor becomes clinically evident only when both are active simultaneously. When one of the mechanisms is suppressed, tremor amplitude becomes markedly reduced ${ }^{19}$.

Our study has significant limitations. Our electrophysiologic assessment was limited to the use of an accelerometers, when it could ideally be complemented by a dual channel surface EMG system, able to document synchronous or rhythmic alternating activation in agonists and antagonists muscle groups. Although this additional electrophysiologic parameter is not pathognomonic, it may increase diagnostic accuracy. Other technical parameters that may interfere with the interpretation of electrophysiological recordings of tremors, are the effects of mechanical and postural reflex components and artifacts. To minimize these potential interferences, the use of a mechanical damping device, such as weighting the limb is recommended, as the frequency of the mechanical components tends to be reduced with this method. Unfortunately, this strategy was not used on our study. Finally, the possibility of the co-existence of ET and PD tremor in the same subject can occur in occasional instances. The association of both has been the subject of heated debate in the literature, including evidence of PD pathology in cases with an undisputed diagnosis of ET and the description of cases of ET flourishing into classic parkinsonian syndrome ${ }^{20}$. The current consensus, however, is that the basis of this association is inaccurate and the result of a series of biases, as suggested by rigorous case-control studies showing lack of association or genetic link between ET and $\mathrm{PD}^{21}$. We tried to minimize this possibility by carefully selecting our cases based on validated criteria, excluding those with dubious phenotypes, however this confounding situation cannot be absolutely discarded. 
In summary, the use of tremor analysis in cases of ET and PD does not exclude the need for an adequate clinical assessment as, individually is it not able to differentiate these disorders, with significant overlap of electrophysiological findings. Additionally, we were able to demonstrate three different tremor profiles in PD and show that the combination of rest and action tremor is the most common phenotype.

\section{References}

1. Baumann CR. Epidemiology, diagnosis and differential diagnosis in Parkinson's disease tremor. Parkinsonism Relat Disord 2012;18(Suppl):S90-S92.

2. Munhoz RP, Werneck LC, Teive HA. The differential diagnoses of parkinsonism: findings from a cohort of 1528 patients and a 10 years comparison in tertiary movement disorders clinics. Clin Neurol Neurosurg 2010;112:431-435.

3. Zeuner KE, Deuschl G. An update on tremors. Curr Opin Neurol 2012;25:475-482

4. Burne JA, Hayes MW, Fung VS, Yiannikas C, Boljevac D. The contribution of tremor studies to diagnosis of parkinsonian and essential tremor: a statistical evaluation. J Clin Neurosci 2002;9:237-242.

5. Milanov I. Clinical and electromyographic examinations of Parkinsonian tremor. Parkinsonism Relat Disord 2000;6:229-235.

6. Hoehn MM, Yahr MD. Parkinsonism: onset, progression and mortality. Neurology 1967;17:427-442.

7. Elble RJ. Diagnostic criteria for essential tremor and differential diagnosis. Neurology 2000;54(Suppl):S2-S6.

8. Hughes AJ, Daniel SE, Kilford L, Lees AJ. Accuracy of clinical diagnosis of idiopathic Parkinson's disease: a clinico-pathological study of 100 cases. J Neurol Neurosurg Psychiatry 1992;55:181-184.

9. Cohen O, Pullman S, Jurewicz E, Watner D, Louis ED. Rest tremor in patients with essential tremor: prevalence, clinical correlates, and electrophysiologic characteristics. Arch Neurol 2003;60:405-410.

10. Rajput AH, Rozdilsky B, Ang L, Rajput A. Significance of parkinsonian manifestations in essential tremor. Can J Neurol Sci 1993;20:114-117.

11. Louis ED, Levy G, Côte LJ, Mejia H, Fahn S, Marder K. Clinical correlates of action tremor in Parkinson disease. Arch Neurol 2001;58:1630-1634.
12. Kraus $\mathrm{PH}$, Lemke MR, Reichmann $\mathrm{H}$. Kinetic tremor in Parkinson's disease-an underrated symptom. J Neural Transm 2006;113:845-853.

13. Forssberg H, Ingvarsson PE, Iwasaki N, Johansson RS, Gordon AM. Action tremor during object manipulation in Parkinson's disease. Mov Disord 2000;15:244-254.

14. Lance JW, Schwab RS, Peterson EA. Action tremor and the cogwheel phenomenon in Parkinson's disease. Brain 1963;86:95-110.

15. Schrag A, Schelosky L, Scholz U, Poewe W. Reduction of Parkinsonian signs in patients with Parkinson's disease by dopaminergic versus anticholinergic single-dose challenges. Mov Disord 1999;14:252-255.

16. Deuschl G, Bain P, Brin M. Consensus statement of the movement disorder society on tremor. Ad Hoc Scientific Committee. Mov Disord 1998;13:2-23.

17. Munhoz RP, Espay AJ, Morgante F, et al. Long-duration Parkinson's disease: role of lateralization of motor features. Parkinsonism Relat Disord 2013;19:77-80.

18. Bergman H, Deuschl G. Pathophysiology of Parkinson's disease: from clinical neurology to basic neuroscience and back. Mov Disord 2002;17(Suppl):S28-S40.

19. Marsden CD, Obeso JA. The functions of the basal ganglia and the paradox of stereotaxic surgery in Parkinson's disease. Brain 1994;117:877-897.

20. Labiano-Fontcuberta A, Benito-Leon J. Essential tremor and Parkinson's disease: are they associated? Rev Neurol 2012;55:479-489.

21. Cleeves L, Findley LJ, Koller W. Lack of association between essential trtemor and Parkinson's disease. Ann Neurol 1988;24:23-26. 